\title{
Enseñanza de la medicina legal en el contexto del Espacio Europeo de Educación Superior
}

\author{
Eloy Girela-López
}

\section{Introducción}

La medicina legal es una disciplina que pertenece a la rama pública de la medicina (no es clínica ni privada), pues su intervención trasciende más allá de los intereses privados de un individuo. El médico legista actúa en interés de la sociedad porque el análisis de cualquier accidente, intoxicación, lesión, capacidad mental de un delincuente o incluso la muerte, no son sólo hechos biológicos que afecten en exclusiva a quien los padece. Además de eso, constituyen unos acontecimientos jurídicos con una clara repercusión social: han de someterse a la acción de la justicia para restablecer el equilibrio social que se ha alterado y para que se reparen los daños producidos. Este es el campo de actuación de la medicina legal y forense y del perito médico.

La medicina legal, judicial o forense, cuyas denominaciones sinónimas son las más frecuentes, nació con las exigencias de la justicia, a la que debe quedar indisolublemente ligada, tanto por su naturaleza como por su contenido. Vino a la luz cuando una ley requirió taxativamente la intervención de los médicos como peritos, se modificó y amplió de acuerdo con el derecho codificado y se ha desarrollado a la par que él.

Situada en la encrucijada entre la medicina y el derecho, sirve de nexo de unión entre estas ramas del saber tan dispares. La medicina, hija de la biología, es vacilante, indecisa, perpleja, ya que su misma esencia es una incógnita: la vida. La verdad biológica lleva siempre un mucho de reserva, de incertidumbre, de duda e incluso de impotencia. Por el contrario, el derecho, aun con muchas limitaciones, es más preciso, conciso, justo, su verdad está encuadrada en los códigos y textos, es por tanto una verdad elaborada por el hombre y comprensible en todo momento. Su campo de acción es sumamente amplio, entra en juego siempre que la materia biológica se convierte en sustrato de normas de dere- cho o de disposiciones administrativas de cualquier índole. Es más, su condición de rama especializada de la medicina surge en esta traducción que del hecho biológico realiza para los fines de la justicia.

Son dos, pues, las misiones de la medicina legal y con ellas como ingrediente se han confeccionado muchas definiciones:

- La necesidad de conocimientos clínicos y biológicos para resolver ciertos problemas jurídicos que, de otra manera, quedarían insolubles.

- La función doctrinal que la medicina aporta a la jurisprudencia, al proporcionar conocimientos de naturaleza biológica y médica en el continuo desenvolvimiento y perfeccionamiento del derecho codificado.

Gisbert-Calabuig [1] ya definió la medicina legal como 'el conjunto de conocimientos médicos y biológicos necesarios para la resolución de los problemas que plantea el derecho, tanto en la aplicación práctica de las leyes como en su perfeccionamiento y evolución'

En la transición a los nuevos planes de estudios derivados del plan de Bolonia en el Espacio Europeo de Educación Superior (EEES), la Universidad de Córdoba se implicó en un proyecto piloto para adaptar las nuevas formas de enseñanza. Sin querer entrar a fondo en lo que supone el cambio en los nuevos planes de estudios, una de las principales modificaciones que el EEES pretende impulsar es un enfoque más plural de la actividad docente, dando un mayor peso a otras modalidades presenciales y potenciando especialmente las no presenciales, con el fin de que el estudiante tenga más oportunidades de ser el protagonista en la búsqueda del conocimiento [2].

El camino aún está por recorrer, aunque en cualquier caso, además de armonizar las enseñanzas de la medicina legal en las facultades de medicina, habría que armonizar la propia sustantividad de los
Facultad de Medicina. Universidad de Córdoba. Córdoba, España.

Correspondencia: Prof. Eloy Girela López. Facultad de Medicina. Universidad de Córdoba. Avda. Menéndez Pidal, s/n. E-14004 Córdoba.

E-mail:

ft1gilpe@uco.es

(c) 2011 Educación Médica 
contenidos de esta especialidad en el ámbito europeo. Por lo que respecta a la medicina legal, como rama social de la medicina se ve afectada aún más que el resto de las especialidades médicas individuales o clínicas por los cambios sociales y las diferencias existentes entre los distintos países. Esta circunstancia, ya destacada en la Declaración de Bolonia [3], influye de manera especial en nuestra disciplina al quedar plasmada en las diversas legislaciones y regulaciones de cada estado, que se toman como referencia y marco de actuación práctica para el ejercicio médico-legal.

Aunque hay versiones intermedias, los modelos de la visión de la disciplina oscilan entre los países del norte de Europa, donde en general se identifica la medicina legal y forense con la patología forense casi exclusivamente, y los países mediterráneos, donde quienes se dedican a esta disciplina abarcan todos los posibles campos de actuación, en lo que el profesor Fiori ha denominado muy gráficamente 'síndrome de Leonardo da Vinci'.

Desde mi punto de vista, no hay que renunciar a los contenidos que pueden corresponder a nuestra disciplina o especialidad, aunque hay que ser conscientes de las limitaciones que de forma forzosa han de imponerse. No es prudente pretender un saber enciclopédico, pues al menos en teoría, los campos de actuación de la medicina legal son tantos como los de la propia medicina. Por ello, y tras una formación sólida en las principales materias de nuestra especialidad, se debería tender a la subespecialización en algunas de las principales ramas de la medicina legal y forense, como patología, clínica o laboratoriotoxicología, y todo ello en el seno de institutos integrados que, además de dedicarse al trabajo rutinario de casos periciales, tuvieran una dedicación importante a la docencia y la investigación. Coincido con la opinión de Madea y Saukko [4], dos cualificados expertos en medicina forense, quienes desde la experiencia de su trabajo en países del norte de Europa opinan que el tener trabajos rutinarios periciales, junto con la docencia y la investigación centralizados en un instituto, garantizan que el trabajo diario puede realizarse siempre dentro de estándares científicos de primer nivel. Además, las nuevas cuestiones que van surgiendo en la práctica pueden responderse con una investigación sistemática. Esto es el ideal en cuanto a la investigación en medicina legal y forense: responder cuestiones abiertas desde el trabajo diario, incluso viejas cuestiones, con la tecnología actualmente disponible. Ellos opinan que reduciendo la medicina forense a la patología forense se corre el riesgo de perder el perfil académico para nuestra disciplina. Por último, lanzan el mensaje siguiente:
- Las periciales rutinarias constituyen justamente la base para la docencia y la investigación, pero no pueden ser el soporte por sí solas en el contexto universitario.

- La investigación debe tener prioridad sobre los casos periciales de rutina.

- La necesidad de aportación de fondos que ayuden a financiar la investigación por parte de los Organismos que, precisando de la medicina legal, no participan en la investigación.

El modelo tradicional del ejercicio de la medicina legal en nuestro país, vinculado de manera casi exclusiva a la Administración de Justicia, es insuficiente para una aplicación integral y armónica con el resto de especialidades y legislaciones internacionales, y la dilución de la medicina legal dentro de las especialidades clínicas será insuficiente para su desarrollo con todas las peculiaridades que la caracterizan; por ello es fundamental la conceptualización y definición de un modelo más amplio que no vincule su identidad al contexto donde se aplica.

Contemplar la medicina legal desde la perspectiva de los institutos universitarios médico-legales es una praxis empleada en muchos países europeos (Italia, Alemania, Dinamarca, Suecia, etc.) desde hace años. Es una medicina legal integrada, coherente y coordinada por profesores universitarios que trabajan simultáneamente en la resolución de los problemas periciales para la Administración de Justicia. El camino que nuestro país trazará está aún por recorrer, aunque puede predecirse que los institutos, concebidos bajo el organigrama del Ministerio de Justicia -de sus respectivas consejerías en las comunidades autónomas-, no parecen contar con los profesores universitarios para que constituyan uno de sus pilares. De hecho, aunque el reglamento de estos institutos [5] es un tanto ambiguo, sólo está previsto que los profesores formen parte de la ' $\mathrm{Co}$ misión de Docencia e Investigación' y no se ha articulado ninguna figura para que pueda compatibilizarse el ejercicio de la docencia con la medicina forense, de forma análoga a como sucede con los profesores de materias asistenciales que trabajan para el Sistema Nacional de Salud [6]. De forma igual de lamentable, tampoco los médicos forenses que trabajan en el seno de los institutos de medicina legal pueden acceder a los cuerpos docentes numerarios de las universidades, como consecuencia de la ley de incompatibilidades del personal al servicio de las administraciones públicas [7], aunque en este último caso al menos sí pueden ser profesores asociados, que es lo que sucede en algunas universidades como la de Córdoba, a través de la firma de conve- 
nios. Ambas circunstancias dificultan una docencia integrada y de calidad.

Por si fuera poco, desde el Ministerio de Sanidad y Política Social tampoco se presta la atención que merece a la especialidad de medicina legal y forense. A día de hoy, a pesar de que el acceso a dicha especialidad es vía MIR, es una especialidad de 'escuela' en donde no se percibe retribución alguna durante el periodo de formación, sino que incluso se tiene que abonar una matrícula. Además, al finalizar el periodo formativo, los especialistas tampoco encuentran fácilmente un campo de trabajo, pues no se exige la especialidad para ejercer la actividad pericial en nuestro país ni tampoco el Ministerio de Justicia la exige para ingresar en el cuerpo de médicos forenses, desaprovechando a unos profesionales formados. En conjunto, un panorama poco alentador si nuestros políticos y autoridades con responsabilidad en este campo no aportan algo de cordura.

Entre las muchas consecuencias que se derivan de la conceptualización 'judicial' de la medicina legal, sin duda, una de las que más se ha visto afectada ha sido la calidad de la pericia, tanto por el modelo adoptado hasta la actualidad - a pesar de la entrada en funcionamiento de institutos de medicina legal- en todas las comunidades autónomas, como por las referencias y criterios adoptados por los profesionales (médicos forenses) a la hora de desarrollar sus funciones, así como por las especiales características del contexto en que se valoraba el resultado de la pericia.

Esta situación y el desarrollo del trabajo médicoforense en un mundo muchas veces ajeno a los criterios científicos que deben regir el desarrollo de una disciplina, ha facilitado que el médico forense se identificara con la figura de autoridad propia del mundo judicial, y que sus informes y consideraciones vinieran respaldadas más por su posición que por su argumentación científica, con lo cual la calidad de la pericia individual y del modelo de la medicina forense se ha visto afectada de manera significativa.

Un nuevo modelo de medicina legal conlleva una ampliación del concepto tradicional de la medicina legal como disciplina vinculada de manera estrecha, casi exclusiva, a la justicia para situarla, también como rama social de la medicina, alrededor de los problemas de salud pública.

\section{Objetivos y contenidos de la asignatura de "Medicina Legal"}

El aprendizaje debe concebirse como 'un proceso continuo de adquisición del hábito de la competen- cia profesional', en este caso de la competencia ('lo que se es capaz de hacer') en el ámbito de la medicina legal como médico general. Este proceso pasa por la adquisición de diferentes niveles de competencia que exigen la definición de objetivos determinados, el diseño del método docente apropiado a los objetivos propuestos y una evaluación dirigida a todas las áreas de competencia incluidas en los objetivos de este nivel. En este contexto se suelen distinguir los objetivos institucionales, intermedios y específicos.

Entenderemos como objetivos docentes institucionales los objetivos docentes del currículo completo de la formación de los estudiantes en cada licenciatura, que vienen expresados en las directrices generales propias de cada plan de estudios. Según recoge el decreto de directrices para la elaboración de planes de estudios de las facultades de medicina [8], los objetivos generales de la asignatura de 'Medicina Legal' son los contenidos en la descripción de la asignatura por dichos planes de estudios:

- Conocer los problemas médico-legales del sujeto vivo y del cadáver.

- Tipos y mecanismos de muerte.

- Semiología cadavérica.

- Toxicología forense, industrial y ambiental.

- Aspectos éticos y legales del ejercicio de la medicina.

Los objetivos intermedios expresan en términos generales los conocimientos que se espera que haya adquirido un alumno o alumna al terminar una etapa del proceso educativo con el fin de llevar a cabo ciertas tareas o de continuar su formación y capacitación. Pueden deducirse de los anteriores, y podrían resumirse en:

- Conocer el ordenamiento jurídico vigente en los aspectos en que asuntos médicos o biológicos puedan tener repercusiones legales: mecanismos que dan lugar a lesiones en el ser vivo y en el cadáver, tipos de lesiones y trascendencia jurídica de éstas, delitos de lesiones, contra la libertad sexual y problemas de psiquiatría forense en los campos penal y civil.

- Capacitar al alumno para que utilice y redacte convenientemente los principales documentos médico-legales.

- Conocer los tipos y mecanismos de muerte, y manejar correctamente la semiología cadavérica. Implicaciones legales y sociales de la muerte. Trasplantes de órganos. Poseer los conocimientos teórico-prácticos que permitan al alumno la realización de una autopsia reglada y la toma de muestras para un diagnóstico post mortem. 
- Poder reconocer un caso de intoxicación y aplicar las medidas terapéuticas de urgencia. Ha de saber solicitar las investigaciones analíticas oportunas y resolver los problemas médico-legales que se plantean.

- Proporcionar a los futuros médicos un nivel de formación suficiente sobre los aspectos éticos, deontológicos y legales imprescindibles para su ejercicio profesional.

Los objetivos específicos corresponden a la actividad de aprendizaje y han de ser pertinentes, concretos, realizables y mensurables. Se trata en esencia de puntualizar de antemano el fruto que se espera obtener de la labor docente, cualquiera que sea la técnica didáctica empleada. En la práctica corresponderían a los objetivos concretos a conseguir en cada tema de la asignatura y que en definitiva vienen determinados por el contenido de ésta.

Desde mi punto de vista, los objetivos específicos podrían resumirse en los siguientes:

- Capacitar al alumno para que redacte y utilice convenientemente los siguientes documentos médico-legales: partes, recetas, oficios, certificados, informes y declaración, y todos aquellos requeridos en las relaciones con las autoridades sanitarias y con la Administración en general.

- Capacitar al alumno para que pueda llevar a cabo un diagnóstico médico-legal correcto de los diferentes tipos de lesiones, tanto vitales como post mortem.

- Hacer que el alumno conozca la trascendencia y las obligaciones legales que se derivan de la existencia de cualquier lesión, así como la de ponerlas en conocimiento de instancias judiciales.

- Aunque cada vez con menor probabilidad de tener que enfrentarse a la práctica necrópsica, el alumno ha de poseer los conocimientos teóricoprácticos indispensables para poder practicar una autopsia judicial en el caso de ser requerido para ello.

- El alumno debería conocer todos aquellos aspectos de la legislación que hacen referencia al ejercicio de la medicina y las posibles implicaciones legales relacionadas con su ejercicio que pueden dar lugar a reclamaciones de responsabilidad profesional, a actuaciones como perito o testigo, etc.

- Interiorizar los valores éticos y los principios deontológicos que rigen el ejercicio de la profesión, respetando especialmente el principio de autonomía del paciente y el derecho al consentimiento informado y al secreto profesional.

- Capacitar al alumno para que pueda llevar a cabo un diagnóstico médico-legal correcto de los prin- cipales trastornos psicopatológicos, en base al establecimiento de la imputabilidad, capacidad civil, estado de drogodependencia, intoxicación o síndrome de abstinencia, etc., y relacionando esta patología con las implicaciones legales de las normas que la regulan.

- El alumno ha de saber reconocer un caso de intoxicación y solicitar las investigaciones analíticas oportunas. Ha de saber aplicar la terapéutica adecuada y resolver los principales problemas médico-legales que plantea un cuadro de intoxicación.

- Transmitir los conocimientos teórico-prácticos necesarios sobre el valor del laboratorio médicolegal en el estudio de muestras biológicas implicadas en el diagnóstico de los indicios criminales, investigación de la paternidad, etc. Con especial énfasis tendrá que conocer cómo hay que buscar y recoger dichos indicios para que no se entorpezca la investigación.

No obstante la enunciación realizada de los objetivos específicos, una concepción pedagógica de dichos objetivos permite clasificarlos del siguiente modo:

\section{Del área cognitiva}

Comportan la adquisición de conocimientos o de información, siendo necesario ejercitar la memoria, la comprensión, el análisis y la síntesis de los hechos $y$, finalmente, la resolución de problemas.

En el caso particular de la medicina legal y forense, en etapas no muy lejanas, el único objetivo de un curso era la adquisición de información, limitando las técnicas del proceso educativo al uso de la lección magistral. En todo caso, y aun teniendo en cuenta la modificación constante de los conocimientos médico-legales y la limitación del tiempo de profesores y alumnos, es necesario desarrollar un programa de medicina legal y forense con la máxima información posible, pero sobre todo que sea básica para el desarrollo de posteriores etapas del currículo.

Según el Libro blanco del título de Grado en Medicina, elaborado en el año 2005 por la Conferencia Nacional de Decanos de las Facultades de Medicina [9], los objetivos cognitivos ('lo que hay que saber') específicos propuestos en el bloque de 'valores profesionales y ética médica, en lo referente a la medicina legal, son los que se expresan en la tabla I.

Asumo en su práctica totalidad los objetivos anteriormente mencionados, si bien matizaría los objetivos 14 (genética forense) y 19 (ecotoxicología). En concreto, creo que los conocimientos de genética forense exigibles para el perfil del médico gene- 
ral han de ser muy elementales, si acaso deben saber algunos, y en referencia al tema de la ecotoxicología creo que no es apropiado el término o no son apropiados los objetivos. A lo sumo, habría que hablar de toxicología ambiental (no ecotoxicología), y en especial o en exclusiva de aquella que, en el medio ambiente laboral o doméstico, puede provocar alteraciones en la salud del hombre.

\section{Del área sensitivomotriz}

Su finalidad es lograr la adquisición de una habilidad práctica ('lo que hay que saber hacer'), lo cual se consigue mediante la información de los fundamentos teóricos de dicha habilidad o técnica, la imitación, manipulación, coordinación y repetición de determinadas acciones.

En el caso particular de un curso general de medicina legal y forense, el alumno debe conocer las técnicas y los métodos fundamentales utilizados en la investigación médico-legal y, muy concretamente, los aspectos relacionados con el método pericial.

En cualquier caso, los conocimientos prácticos han de reforzar los conocimientos teóricos adquiridos y el alumno debe haberse familiarizado con el manejo de instrumentos y técnicas generales del método científico (diseño de experimentos, adquisición de datos, elaboración, presentación y tratamiento estadístico de resultados, crítica y discusión de éstos, etc.), así como con su aplicación a los problemas prácticos que se plantean ante la medicina legal.

Según el anteriormente mencionado Libro blanco del título de Grado en Medicina [9], los objetivos específicos del área sensitivomotriz son los que se expresan en la tabla II.

\section{Del área afectiva}

Persiguen la creación de actitudes que llevan a un estado de receptividad frente a ciertas solicitudes, consiguiendo respuestas adecuadas a ella, estructurándolas en una escala estable y coherente de valores. Estos objetivos incluyen:

- Reconocer los elementos esenciales de la profesión médica, incluyendo los principios éticos y las responsabilidades legales.

- Comprender la importancia de tales principios para el beneficio del paciente, de la sociedad y la profesión, con especial atención al secreto profesional y al consentimiento informado.

- Saber aplicar el principio de justicia social a la práctica profesional.

- Desarrollar la práctica profesional con respeto a la autonomía del paciente, sus creencias y cultura.
Tabla I. Objetivos cognitivos específicos referentes a la medicina legal [9].

Valores profesionales: excelencia, altruismo,

sentido del deber, responsabilidad, integridad y honestidad

Fundamentos básicos sobre el derecho médico.

Conocer los instrumentos legales del ejercicio de la profesión médica

Pericia profesional. Causas y semiología de las lesiones

Indemnización de daños. Invalideces. Baremos

Responsabilidad profesional

Consentimiento informado

Implicaciones sociales y legales del fenómeno de la muerte. Trasplante de órganos

Evolución normal del cadáver. Diagnóstico post mortem

Conservación del cadáver

Psiquiatría forense

Delitos contra la libertad sexual y el delito de aborto.

Obstetricia y ginecología forense

Medicina del trabajo

Conocimientos elementales de criminalística, del examen del lugar de los hechos, los principales indicios, su revelado, recogida y transporte

Genética forense

Actuación médico-legal en caso de grandes catástrofes

Violencia familiar. Violencia de género. Maltrato al niño y al anciano

Mecanismos básicos de acción de los tóxicos.

Principales agentes causantes de intoxicaciones

Drogas de abuso

Ecotoxicología

Principales documentos médico-legales

- Reconocer las propias limitaciones y la necesidad de mantener y actualizar su competencia profesional.

- Desarrollar la práctica profesional con respeto a otros profesionales de la salud y fomento del trabajo en equipo.

- Adopción del pensamiento crítico: adopción de una actitud inquisitiva y racional ante los procesos, reconocimiento de la falta de certeza derivada de los límites del conocimiento. 
Tabla II. Objetivos específicos del área sensitivomotriz referentes a la medicina legal [9]

\begin{tabular}{|c|c|}
\hline \multirow{3}{*}{$\begin{array}{l}\text { Saber hacer } \\
\text { con competencia } \\
\text { (rutinariamente } \\
\text { y sin supervisión) }\end{array}$} & Leer textos legales e interpretarlos \\
\hline & Redactar correctamente documentos médico-legales \\
\hline & Tomar muestras en un cadáver y prepararlas para su desplazamiento \\
\hline \multirow{4}{*}{$\begin{array}{l}\text { Haberlo practicado } \\
\text { tuteladamente (bajo } \\
\text { supervisión del tutor) }\end{array}$} & Realizar un peritaje e informe médico-legal \\
\hline & $\begin{array}{l}\text { Aplicar medidas elementales de prevención de accidentes } \\
\text { de trabajo y enfermedades profesionales }\end{array}$ \\
\hline & Atención a una víctima de la violencia en el medio doméstico \\
\hline & Investigación criminalística básica \\
\hline \multirow{6}{*}{$\begin{array}{l}\text { Haberlo visto practicar } \\
\text { por un experto }\end{array}$} & Peritaje de valoración de daños personales \\
\hline & Exploración básica de una presunta víctima de un delito contra la libertad sexual \\
\hline & Exploración básica de un paciente en el caso de sospecha de un aborto criminal \\
\hline & $\begin{array}{l}\text { Diagnóstico clínico y de laboratorio en el caso de impregnaciones } \\
\text { por tóxicos frecuentes en nuestro medio laboral }\end{array}$ \\
\hline & Elaborar una ficha dactilográfica y odontológica para la identificación de cadáveres \\
\hline & Autopsia judicial reglada (según el actual ordenamiento legal) \\
\hline
\end{tabular}

\section{Propuestas concretas para los nuevos planes de estudio en el EEES}

Tras las reflexiones esbozadas hasta aquí, y ante las modificaciones que se avecinan para adaptarnos a la convergencia europea en el EEES, me permitiré hacer alguna propuesta concreta sobre la enseñanza a impartir en el programa docente de medicina legal.

El uso de nuevas tecnologías al servicio del estudiante se hace imprescindible. El desarrollo de los contenidos de la asignatura en un aula virtual que permita el acceso telemático posibilita colocar contenidos que refuercen y complementen lo explicado en las horas presenciales de teoría. En dicha plataforma virtual se produce una comunicación muy fluida con los alumnos, que pueden consultar dudas en un foro específico, realizar tutorías virtuales, recibir de forma eficaz anuncios o citaciones que el profesor deba comunicarles, visionar los temas explicados en clase con las imágenes correspondientes, realizar ejercicios prácticos que se les proponen (corregidos y comentados luego por el profesor), acceder a información complementaria que se les selecciona (enlaces a páginas web, vídeos o artículos científicos), realizar exámenes de autoevaluación, etc. Por todo ello, el uso de esta herramienta será de extraordinaria importancia para el aprendizaje del alumno. También se convierte en una herramienta que ayuda a la tarea de evaluación de la enseñanza que debe realizar el profesor, pues registra y permite consultar de forma rápida y exhaustiva toda la actividad realizada por los alumnos a lo largo del curso.

En lo que respecta a las prácticas que realizan los alumnos, hay que tratar de potenciarlas todo lo posible. Dada la actual organización de la medicina legal en nuestro país (articulada fundamentalmente por las pericias oficiales de los médicos forenses en los institutos de medicina legal), no hay otra forma de hacer esto que posibilitando que los alumnos acudan a estos institutos y acompañen a los médi$\cos$ forenses en su actividad diaria para que vean, aprendan e imiten las habilidades necesarias que puedan servirles en un futuro. Así, la Universidad de Córdoba firmó un convenio con la Consejería de Justicia y Administración Pública de la Junta de Andalucía, el 12 de enero de 2007, que permite que los médicos forenses puedan ser contratados como profesores asociados y que los alumnos puedan realizar allí dichas prácticas, donde acuden a pasar consulta en clínica médico-forense y tienen la oportunidad de aprender:

- Documentos médico-legales.

- Anamnesis y exploración médico-legal de agredidos o lesionados.

- Utilización de baremos para valorar daño corporal.

- Valoración del grado de imputabilidad o incapacidad de personas con trastornos mentales que están envueltas en asuntos con alguna implicación judicial.

- Valoración de problemas específicos de violencia de género.

También presencian la realización de autopsias judiciales para esclarecer las causas y las circunstancias que rodean a las muertes de origen desconocido, o las muertes violentas y sospechosas de criminalidad. En la medida en que puedan ver varias autopsias podrán incluso intervenir en la realización de alguna de ellas.

Por último, los alumnos realizan una mañana de acompañamiento al forense que está en labores 'de guardia, con lo que pueden presenciar el reconocimiento urgente de lesionados, especialmente en casos de violencia de género, el reconocimiento y asistencia al detenido, así como la diligencia de levantamiento de cadáver, asistencia a juicios donde acude como perito el médico forense, etc.

Por otro lado, desde mi punto de vista, existe una descompensación entre la carga teórica y la prácti- 
ca, con una disminución de horas lectivas reales que permitan enseñar los contenidos cognitivos específicos en nuestra asignatura. Ello obliga, aunque sea utilizando otras técnicas didácticas diferentes a la lección magistral (tipo seminarios u otras actividades), a incluir parte del contenido teórico en las horas de los créditos prácticos.

Hay diferentes propuestas en los nuevos planes de estudios del Grado en Medicina para esta asignatura, aunque las distintas opciones se debaten entre las minoritarias de incluir los contenidos de 'Ética Médica' y 'Medicina Legal' en una sola asignatura (como sucede en Salamanca, Lleida y Córdoba), con una carga docente de entre 6 y 9 ECTS, o la postura de la mayoría de las facultades de dividir los contenidos en dos asignaturas: por una parte, 'Medicina Legal', con una carga docente de entre 3 y 9 ECTS, y por otra, 'Ética Médica', con una carga docente de entre 2 y 6 ECTS. En cualquiera de los casos, mi opinión es que hay que potenciar en los programas el peso que se da tanto a los temas de derecho médico como de ética (esta materia se ha incluido como obligatoria en los nuevos planes de estudio). Además, ambas materias se prestan bastante bien a sesiones presenciales tipo seminarios, en los que el profesor puede dirigir a un grupo no muy numeroso de alumnos (entre 15 y 20), para que lean alguna información antes de que se celebre la sesión presencial y luego participen activamente con sus opiniones y preguntas. En concreto, en este tipo de formato imparto actualmente temas sobre consentimiento informado (dos horas presenciales), secreto profesional (una hora presencial), eutanasia (dos horas presenciales) y responsabilidad profesional médica (dos horas presenciales). Al final de cada seminario se exponen supuestos casos prácticos reales para su discusión y se propone algún otro para su realización posterior por los alumnos (individualmente $o$ en grupos muy reducidos de dos o tres) como tarea (en cada caso, conlleva un trabajo como actividad académicamente dirigida no inferior a dos horas de trabajo individual).

Otros temas se pueden impartir combinando teoría y práctica, de forma que se refuerza el aprendizaje de las competencias específicas. En concreto, utilizo este formato en temas de criminalística (una hora presencial de teoría más una hora y media presencial de práctica) y muerte súbita (una hora y media presencial de teoría más una hora presencial de práctica). En el caso de criminalística, tras una breve exposición de la importancia del estudio de los principales indicios biológicos, de la información que pueden suministrar y de las técnicas de recogida y envío al laboratorio, pasamos a practicar una marcha analítica básica de manchas de sangre (cada alumno eluye una costra y realiza pruebas de diagnóstico genérico con cristales de hemocromógeno, de Teichman, test de bencidina y luminol) y una marcha analítica básica de manchas de semen (cada alumno realiza un test de fosfatasa ácida sobre una tela manchada). Esta práctica se imparte en el Aula de Microscopía. En el caso de temas de muerte súbita, tras una exposición de los conceptos de muerte súbita y de las principales causas y epidemiología de la muerte súbita en el niño y en el adulto, se explica detenidamente la técnica de disección cardiaca y se practica dicha técnica por parte de los alumnos en grupos muy reducidos (3-4 alumnos) utilizando corazones de cerdo. Esta práctica se imparte en la Sala de Disección.

\section{Bibliografía}

1. Gisbert-Calabuig JA. Medicina legal y toxicología. Valencia: Saber; 1977.

2. De Miguel M. Métodos y modalidades de enseñanza en la educación superior. In De Miguel M, coord. Metodologías de enseñanza y aprendizaje para el desarrollo de competencias. Orientaciones para el profesorado universitario ante el Espacio Europeo de Educación superior. Madrid: Alianza Editorial; 2006. p. 17-26.

3. Association for Medical Education in Europe and the World Federation for Medical Education. Statement on the Bologna process and medical education. Copenhagen/Dundee: AMEE/WFME; 2005.

4. Madea B, Saukko P. Future in forensic medicine as an academic discipline -focusing on research. Forensic Sci Int 2007; 165: 87-91.

5. Boletín Oficial del Estado. Real Decreto 386/1996, de 1 de marzo, por el que se regula la estructura y funcionamiento de los institutos de medicina legal.

6. Boletín Oficial del Estado. Real Decreto 1558/1986, de 28 de junio, por el que se establecen las bases generales del régimen de conciertos entre las universidades y las instituciones sanitarias.

7. Boletín Oficial del Estado. Ley 53/1984, de 26 de diciembre, de incompatibilidades del personal al servicio de las Administraciones Públicas.

8. Boletín Oficial del Estado. Real Decreto 1417/1990 y siguientes, por los que se establecen los títulos universitarios de licenciados y las directrices de los planes de estudio.

9. Peinado-Herreros JM, coord. Libro blanco del título de Grado en Medicina. Granada: Agencia Nacional de Evaluación de la Calidad y Acreditación; 2006. 